\title{
Expectations for contrast-enhanced ultrasonography
}

\author{
Nakashima Kazutaka
}

(C) The Japan Society of Ultrasonics in Medicine 2019

Whereas dynamic contrast-enhanced studies are the mainstay of diagnostic imaging in the case of magnetic resonance imaging (MRI) and computed tomography (CT), morphological diagnosis using B-mode is the mainstay in ultrasonography (US). Of course, Doppler mode is widely used, but a contrast agent is not used in Doppler mode, and as such it currently only allows for simple hemodynamic studies, not dynamic studies. I think further advances in research on contrast agent-based diagnostic approaches are needed going forward in order for US to become a trustable diagnostic imaging modality like MRI and CT.

In the case of contrast-enhanced US using Sonazoid, the contrast agent does not leak from blood vessels, making it possible to purely assess the vascular bed (contrast harmonic image) and evaluate dynamic pure blood flow, including minute tumor neo-vascularity (contrast Doppler image (SMI, DFI, MVI)). Observation of these hemodynamics is not possible with pathological specimens that have shrunk as a result of the blood run-out and its fixing process. Hence, contrast-enhanced US is thought to be the only one tool that can investigate the true tumor vasculature.

From the standpoint of current practice, Sonazoid-based US is covered by insurance for examinations targeting the liver and breast. In fact, a technique for diagnosing severity in the liver has already been established, with the diagnostic accuracy for liver masses surpassing that of dynamic MRI. One could argue that that it has become a sufficiently standard examination.

In the case of breast US, however, a dynamic study of breast masses has not been nearly as well established, to say nothing of its widespread adoption.

Why has not it been widely adopted despite the absence of any issues associated with its clinical use? US has traditionally been thought of as a simple and safe examination

Nakashima Kazutaka

urbandoc@med.kawasaki-m.ac.jp

1 Department of General Surgery, Kawasaki Medical School, Okayama, Japan that can be performed by a technician, so perhaps contrastenhanced US appears to be a high hurdle to overcome. It is true that contrast-enhanced US is more complex, requiring a consent form and infusion of a contrast agent by a doctor or nurse, but Sonazoid itself is an extremely safe agent with almost no risk, with the exception of patients with egg allergies, and it can be stored at room temperature, so it can always be kept on hand in the laboratory and outpatient clinic. With a little ingenuity, it is not that difficult to easily perform an examination.

What is likely needed is for contrast-enhanced US to generate more added value. If so, demonstrating and generating awareness of the advantages of contrast-enhanced US may be required for its widespread adoption.

Several findings were reported at last week's Annual Scientific Meeting of the Japan Society of Ultrasonics in Medicine. First, it was reported that contrast-enhanced US was useful for determining the extent of resection of breast cancer. It was also reported that contrast-enhanced US was useful for interim assessment of preoperative chemotherapy, and that it predicts the pathological assessment of therapeutic response. In addition, it was reported that it was possible to distinguish between tumor neo-vascularity and dilated normal vessels in and around tumors using contrastenhanced US, and that it may be possible to evaluate the true hemodynamics, which is not possible with conventional pathological examinations.

These reports indicate that research on breast contrastenhanced US and research on adding clinical value are showing progress, and they give us hope that contrastenhanced US will be more widely adopted in the future in the field of breast imaging. 